\title{
DLR VR-SCAN: A Versatile and Robust Miniaturized Laser Scanner for short range 3D-Modelling and Exploration in Robotics
}

\author{
Simon Kielhöfer, Thomas Bahls, Franz Hacker, Tilo Wüsthoff and Michael Suppa
}

\begin{abstract}
Precise and robust perception of the environment is crucial for highly integrated and autonomous robot systems. In this paper the dedicated design of a triangulation based laser range scanner optimized for 3D-modelling and autonomous exploration in robotics is presented. The presented laser scanner design is based on an extremely small MEMS scan head permitting a compact, lightweight and highly integrated implementation allowing for hand-eye operation. Special capabilities like variable range and confidence rating of the measuring values increase robustness. The design considerations and a prototype are described and experimental results are presented.
\end{abstract}

\section{INTRODUCTION}

Robot perception requires robust and versatile sensors. Robustness is crucial to execute tasks in unknown environments. Versatility requirements arise from the variability of different perception tasks, such as object modelling and recognition, visual servoing, exploration, and collision avoidance. Many different sensors nowadays exist, they can be classified by accuracy, field of view (FOV), frame rate, etc. in order to determine their suitability for applications, i.e. stereo vision, structured-light sensors, time-of-flight sensors, to name a few. In most cases, the size of the sensor is important, especially when mounted on the robots end-effector or when used on mobile robots. The variety of perception requirements can hardly be met by one single sensor. One step to increased versatility is the multi sensory approach described in [1]. Complementary to this, task restrictions can also be reduced by more versatile sensor design specified to certain fields of applications.

Versatile sensors can provide solutions for complex applications. The sensor development presented in this paper addresses the application of autonomous modelling using a robot. The sensor is mounted on the end-effector in the "eye-in-hand" setup (Fig. 1). NB that the sensor is not limited to this application, multiple others exist [2] [3]. It is assumed that the classical sense-plan-act method is applied. Furthermore, the scene is a priori unknown. The requirements on the sensor are as follows: robust determination of safe-for-motion (SFM) areas of the robot, i.e. exploration of the environment, and generation of high quality models of objects in the environment for grasp-planning. The dexterity and payload of the robot shall not be constrained, therefore the sensor should be small and light-weight.

For exploration of the robot workspace (e.g. for path planning, collision avoidance) a confidence rating value is required [4], especially for free space measurements in order

All Authors are with the Institute for Robotics and Mechatronics, German Aerospace Center (DLR), Münchner Str. 20, 82234 Weßling, Germany \{firstname.lastname\}edlr.de

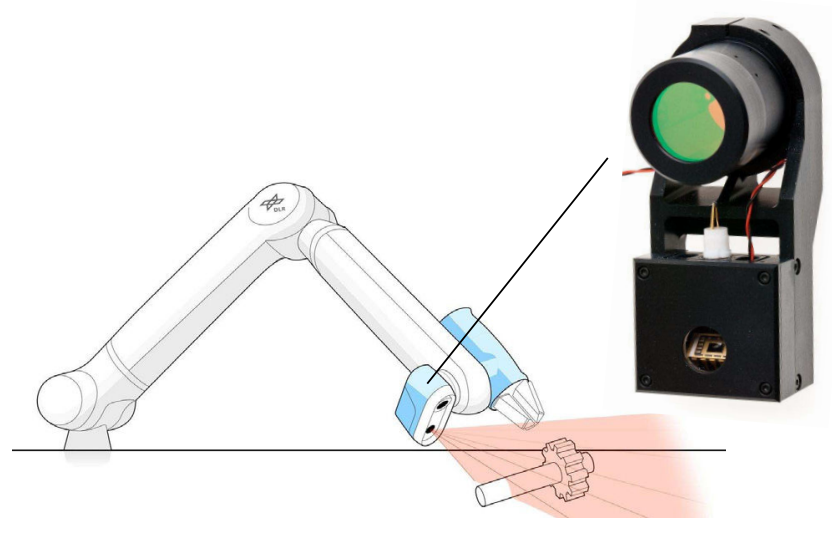

Fig. 1. The VR-SCAN

to be able to safely determine SFM. Further, the measurement range of the sensor must cover the robot work space, while precise short range modelling is demanded at the same time. Most sensors do not provide confidence rating, which is usually compensated by long range vision, e.g. stereo. Short range sensors are well suited for modelling of objects, e.g. stripe measuring principles are advantageous since the robot can extend the acquisition to $3 \mathrm{D}$ by sequentially sweeping across the objects.

Our approach presents a miniaturized versatile sensor prototype (Fig. 1) filling the gap between the requirements of exploration and precise object modelling. This paper continues as follows: First, the State of the Art on sensors is described. Then, the design considerations for the sensor development are elaborated. The calibration of the sensor is described, and the paper is concluded with an outlook on future work.

\section{StATE OF The ART}

In 2004, Blais gave an excellent overview over 20 years of range sensor development, considering both relevant measuring principles and numerous commercial and scientific implementations [5]. Some important sensors are summarized exemplarily in Tab. I: The first group is named modelling systems (MOD), where a plethora of commercial sensors is available today. The laser stripe profiler is the by far most used system, also in science [6]. The easy, cheap, precise and still compact solution is very suitable for modelling on robots. The main drawback is the illumination of the whole line at once, so local reflectance differences in the FOV cannot be respected. That leads to reduced optical signal-to-noise-ratio $(\mathrm{S} / \mathrm{N})$ and does not allow for SFM measurements. Generally these close to mid-range systems 
TABLE I

SENSORS, APPLICATIONS AND CLASSIFICATION

\begin{tabular}{l|l|l|l}
\hline Sensor & Type & Applications & Class \\
\hline \hline ModelMaker $^{\mathrm{a}}$ & LSP & 3D Modelling & \multirow{2}{*}{ MOD } \\
Steinbichler Comet $^{\mathrm{b}}$ & PP & & \\
DLR LSP [6] & LSP & & \multirow{2}{*}{ EXP } \\
\hline Hokuyo SOKUIKI ${ }^{\mathrm{C}}$ [7] & TOF & $\begin{array}{l}\text { Exploration, SLAM, } \\
\text { collision avoidance } \\
\text { Sick Scanners }\end{array}$ & TOF \\
DLR Laser Scanner [8] & SPT & $\begin{array}{l}\text { (CA), (3D Modelling) } \\
\text { (CA }\end{array}$ & \\
\hline
\end{tabular}

Type: $\mathrm{LSP}=$ Laser Stripe Profiler, $\mathrm{PP}=$ Pattern projection, $\mathrm{SPT}=$ Single point triangulation, TOF $=$ Time of flight or phase shift

${ }^{a}$ us.nikonmetrology.com/handheld_scanners ${ }^{b}$ www.steinbichler.de

${ }^{\mathrm{c}}$ www.hokuyo-aut.jp ${ }^{\mathrm{d}}$ www.sick.com

aim at high precision modelling, mostly in combination with very accurate pose reference systems like measuring arms or tracking systems. With the eye-in-hand setup in mind, most commercial systems drop out due to their size and weight in addition.

Passive stereo camera systems are precise and popular offthe-shelf systems, suffering from strong limitations in object texture and illumination. In the recent years increasingly research is done in the field of active $2.5 \mathrm{D}^{1}$ sensors, like in PMD (Photonic Mixer Device, www.pmdtec.com) technology or developments in game industry (Microsofts Kinect, www.xbox.com/en-US/kinect), but precision and confidence are still deficient.

A widespread type of exploration sensors (EXP) are 2D time-of-flight or phase-shift range sensors for navigation. They have up to $360^{\circ} \mathrm{FOV}$ and are widely used in mobile robotics. A very small example is the SOKUIKI Scanner [7]. The range is up to $4 \mathrm{~m}$, but due to the phase shift principle it lacks precision $( \pm 10 \mathrm{~mm} @ 20-1000 \mathrm{~mm})$. Its strength is rather mapping and collision avoidance than accurate modelling. The most interesting feature of the DLR Laser Scanner [8] is that the laser power is regulated online for each single measuring point. This yields a confidence value for robust SFM area exploration. Thus it's classified as an exploration sensor despite fair modelling capabilities. Another early implementation which pushes into a similar direction is described in [9]. The sensor works with synchronized scanning triangulation and already has a CCD imaging device. It aims on large depth of view (100-1000mm) and builds very compact to be mounted on a robot gripper.

Autonomous modelling demands versatile perception from the first two classes in Tab. I, enhanced by consequent confidence rating. This tradeoff is considered in the design of the sensor as described in the following section.

\section{DESIGN CONSIDERATIONS}

\section{A. Requirements}

The accuracy and acquisition speed of the sensor should match with the manipulation (grasping) requirements. Therefrom $0.1 \%$ range precision is desired. The minimum measuring range should be around $250 \mathrm{~mm}$ roughly according to the size of a common gripper, and $1 \mathrm{~m}$ maximum distance assures effective exploration. The data rate must be high enough to gather typical objects to being grasped within reasonable time. One scan should be done at least at video

\footnotetext{
${ }^{1} \mathrm{~A} 2 \mathrm{D}$ data array containing depth information is called $2.5 \mathrm{D}$
}

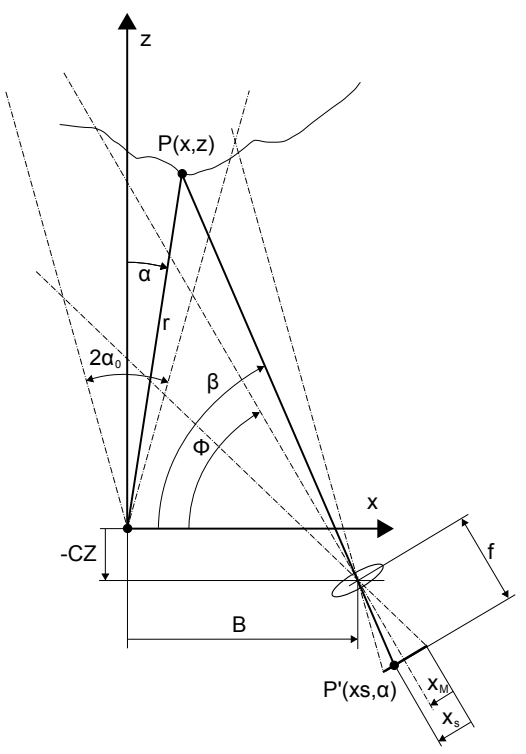

Fig. 2. Geometric Layout and measuring principle of VR-SCAN

frame rate and yield a dense point distribution such that one pan over the object is sufficient. At $300 \mathrm{~mm}$ distance $0.5 \mathrm{~mm}$ point spacing seem adequate (1.7 mrad resolution).

Single point measurement is desired due to lighting immunity and SFM exploration. Regarding the eye-in-hand setup, besides compact sensor dimensions a perception friendly robot design, e.g. with dedicated mechanical and electrical sensor interfaces near the end effector, is advantageous.

\section{B. Measuring Principle}

Since increasing accuracy in the near field is desired, triangulation is the sensor principle of choice. Active 2D triangulation (see Fig. 2) is very precise at close range and features robust data acquisition. At the origin of the sensor coordinate system a laser beam is emitted onto an oscillating mirror and scans within the $(x, z)$-plane the FOV of $2 \alpha_{0}$. The momentarily illuminated point $P(x, z)$ on the object is imaged through a lens on a linear imaging sensor. The point $P^{\prime}$ is represented by the sensors measured variables scan angle $\alpha$ and image coordinate of the peak $x_{s}$. The effective FOV is described by the intersection of scanning field and the FOV of the camera.

\section{Geometric Layout}

The scanner geometry is defined by the base distance $B$, camera bias $\Phi$ and offset $C Z$ and the focal length $f$. The scan amplitude $\alpha_{0}$, the imaging sensor length $l$ and position $x_{M}$ define the FOV (cf. Fig. 2). The layout is optimized for large depth of view from 250 to $1000 \mathrm{~mm}$ (see Tab. II). The scan amplitude is large enough to cover the whole camera FOV in the complete measuring range. The large value for $f$ ensures a high optical resolution but the depth of focus suffers. Thus an autofocus lens has been designed (see Sec. IV-B).

Viewing Fig. 2 there is

$$
z=\frac{x}{\tan (\alpha)}
$$


TABLE II

GEOMETRIC LAYOUT OF VR-SCAN

\begin{tabular}{llll}
\hline base distance & $B$ & 60 & $\mathrm{~mm}$ \\
camera bias & $\Phi$ & 82 & $\circ$ \\
camera offset & $C Z$ & 0 & $\mathrm{~mm}$ \\
focal length & $f$ & 25 & $\mathrm{~mm}$ \\
scan amplitude & $\alpha_{0}$ & 20.5 & $\circ$ \\
CMOS length & $l$ & 14.336 & $\mathrm{~mm}$ \\
\hline
\end{tabular}

and at the same time with the constraint: $\beta<\frac{\pi}{2}$

$$
z=\tan (\beta)(B-x)+C Z \text {. }
$$

Thereby the cartesian dimensions $(x, z)$ can be expressed in sensor coordinates $\left(\alpha, x_{s}\right)$ as

$$
x=\tan (\alpha) z \quad ; \quad z=\frac{\tan (\beta) B+C Z}{1+\tan (\beta) \tan (\alpha)} .
$$

Assuming an ideal lens, $\beta$ results in

$$
\beta=\Phi+\arctan \left(\frac{x_{s}-x_{M}}{f}\right)
$$

with $x_{M}$ representing the position of the imaging sensor w.r.t. the optical axis. When radial lens distortion is considered [10], the corrected image coordinate equals to

$$
\tilde{x}_{s}=x_{s}+k_{1}\left|x_{s}-x_{M}\right|^{3}+k_{2}\left|x_{s}-x_{M}\right|^{5} .
$$

\section{Performance}

1) Angular and radial precision: Assuming $\alpha, C Z=0$ and the imaging sensor axis being parallel to the $x$-axis, the radial and lateral measurement errors $e_{r}$ and $e_{l}$ can be approximated as

$$
e_{r}=r^{2} \frac{p_{p}}{f B}, \quad e_{l}=e_{r} \frac{B}{r}=r \frac{p_{p}}{f},
$$

where $p_{p}$ is the precision of the $x_{s}$-measurement, mainly limited by the peak detection accuracy (see Sec. III-E) and speckle noise. After all the angular scan precision $p_{a}$ should not have larger effect on the lateral error as $p_{p}$, hence it has to satisfy

$$
p_{a} \leq \arctan \left(\frac{e_{l}}{r}\right) .
$$

According to $0.1 \%$ maximum radial error at far range $(1 \mathrm{~m})$, the precision $p_{p}$ has to be $1.5 \mu \mathrm{m}$ and thus the scan angle position error less than 0.06 mrad.

2) Acquisition speed: The requirements lead to a minimum point rate of $22 \mathrm{kHz}$, while full scans should be gathered in video frame rate $(>50 \mathrm{~Hz})$. A non whole-number ratio of camera and scan frequency can enhance the angular resolution trough interlacing.

\section{E. Peak Detection}

There are many approaches to sub-pixel peak detection, summarized in [11]. The needed precision is $\approx 0.2$ pixels $(1.5 \mu \mathrm{m})$ with the $2 \mathrm{~K}$ resolution sensor described in Sec. IVB. Aware of the long focal length and the resulting small depth of focus, mainly blurred images of the measuring point are to be expected. Thus the peaks differ strongly in width and height. Therefore a robust algorithm to detect
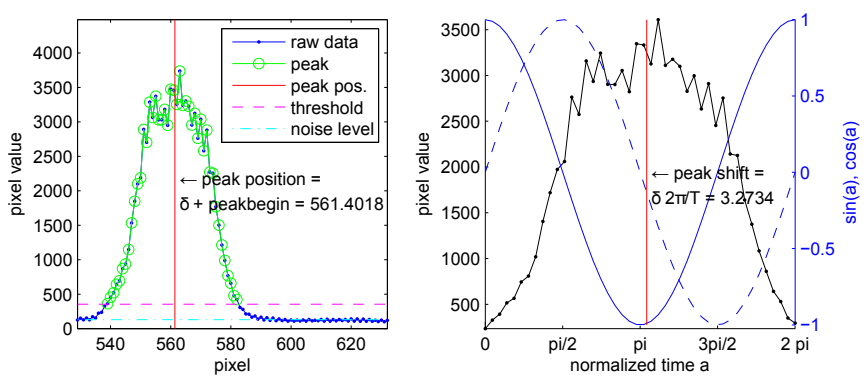

Fig. 3. Fourier-Descriptor Peak Detection example: peak segmentation (left), peak shift determination (right)

reproducible peak positions should evaluate preferably all information contained in the complete peak form, allowing also for low $\mathrm{S} / \mathrm{N}$, saturated and deformed images due to speckle effects.

The method used here, a Fourier-Descriptor based phase detector method, complies with these constraints. The measurement is determined valid if the peak exceeds the minimum S/N snr min. Then the whole peak $\vec{p}$ from the global maximum down to a threshold above noise level is segmented and evaluated, yielding variable peak widths (see Fig. 3 (left)). Let $N$ be the window size, then $T=N-1$ appears as the period and $\vec{t}$ as the normalized time vector of the first harmonic. The phase shift of the peak and thus subpixel position shift $\delta$ relative to the windows left edge is calculated as follows:

$$
\begin{array}{rlrl}
\vec{t} & =[0,1, . ., T] \cdot 1 / T & \vec{a} & =2 \pi \vec{t} \\
\vec{c} & =\cos (\vec{a}) & \vec{s}=\sin (\vec{a}) \\
X & =\vec{c} \cdot p^{T} & Y=\vec{s} \cdot p^{T} \\
\delta^{\prime} & =\operatorname{atan} 2(Y, X) & & \\
\delta & =\left\{\begin{array}{lll}
\delta^{\prime} \frac{T}{2 \pi} & \text { if } \delta^{\prime} \geq 0 & \\
\delta^{\prime} \frac{T}{2 \pi}+T & \text { if } \delta^{\prime}<0 &
\end{array}\right.
\end{array}
$$

In Fig. 3 (right) the normalized peak, the first harmonic sine and cosine waveforms, and the resulting peak shift are depicted. Together with the actual laser power $P_{l}$ profounded confidence statements of both object and SFM measurements can be made. The normalized confidence (quality) $C_{o b j}$ of valid measurements is defined as:

$$
C_{o b j}=s n r / s n r_{\max } \mid \quad s n r \geq s n r_{\min }
$$

If no object is measured, the scanned area is determined to be free of obstacles within range with the confidence $C_{s f m}$ :

$$
C_{s f m}=P_{l} / P_{l, \max } \mid \text { snr }<s n r_{\min }
$$

\section{THE VR-SCAN PROTOTYPE}

\section{A. System design}

The image processing of the camera peak and the estimation of the scan angle should happen in real time, allowing for laser power and MEMS amplitude control. Additionally, the focus control and the conversion $\left(\alpha, x_{s}\right) \rightarrow(x, z)$ has to be done in parallel. All processing, synchronization and control is implemented in an FPGA, which is integrated in the scanner electronics. Thus the scanner itself yields calibrated $(\mathrm{x}, \mathrm{z})$ distance values and can be adapted easily 


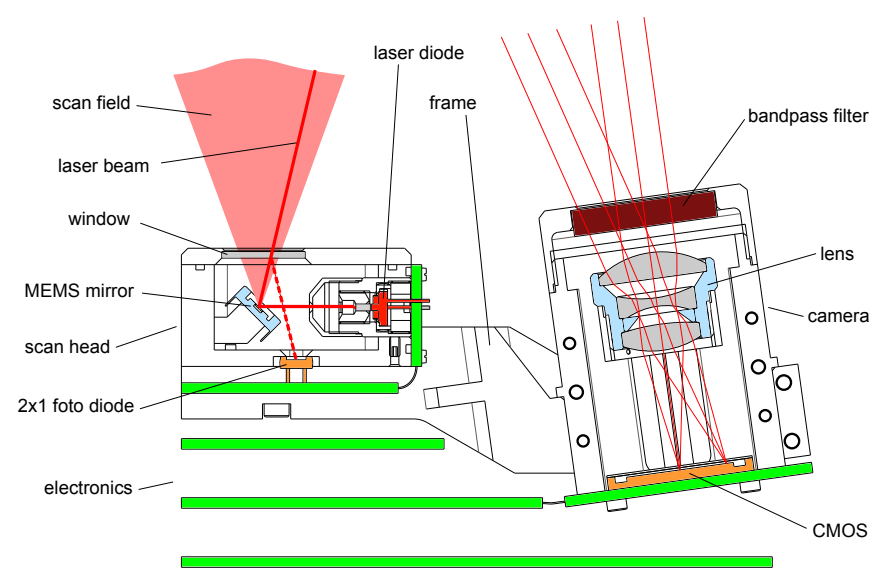

Fig. 4. Cross section trough the scan plane of VR-SCAN

to applications. Moreover, the scanner data interface is kept simple and of low bandwidth and external computing power is not required allowing for mobility.

\section{B. Optics}

The optical components are selected and designed according to the geometrical layout (cf. Tab. II). In particular, the focal length of the lens and size of the linear imaging sensor depend from each other. The chosen 2048 pixels CMOS sensor $^{2}$ is fast at low noise and suitable to this design due to its size and resolution [12]. The CMOS length is 14.336 mm (7 $\mu \mathrm{m}$ square pixels), demanding a lens with $\mathrm{f} \approx 25$ $\mathrm{mm}$. Both small dimensions and a high aperture is desirable. Unfortunately a single lens or achromatic doublet suffer from strong optical aberrations, namely spherical aberration, astigmatism and a high field curvature. Simulations ${ }^{3}$ showed that a triplet eliminates those errors and enhances the illumination of the small pixels of the CMOS significantly due to its planar image field. A very compact lens (f-number 1:3.0) was designed ${ }^{4}$ to cover the needs, see Fig. 4 . The low weight allows the implementation of a small and simple autofocus actuator as described in Sec. IV-E.

A $658 \mathrm{~nm}$ laser diode is collimated by a $\varnothing 1 \mathrm{~mm}$ graded index rod lens with very short focal length. Doing so the laser module and thus the scan head keeps small (Fig. 4). Additionally the beam diameter at the emitting point is narrow allowing for the selected MEMS-mirror device (see IV-C). The laser power is up to $50 \mathrm{~mW}$ which enures a good $\mathrm{S} / \mathrm{N}$ even in bad conditions. The visible wavelength allows for optical feedback to a possibly human operator.

A band pass interference filter with transmission between 645 an $665 \mathrm{~nm}$ keeps the influence of environment light low.

\section{MEMS Scanning Mirror}

A MEMS scanning mirror to deflect the measuring laser beam stands out for its extremely small size and abrasion-free movement [13]. In resonance oscillating mirrors yield high deflection angles both in 1D and 2D mainly for bar code scanners and micro projection displays. For this prototype

\footnotetext{
${ }^{2}$ CMOS 1421 (www.fairchildimaging.com)

${ }^{3}$ using FRED Optical Engineering Software (www.photonengr.com)

${ }^{4}$ Custom design by Linos $\mathrm{GmbH}$, now Qioptiq (www.qioptiq.com)
}

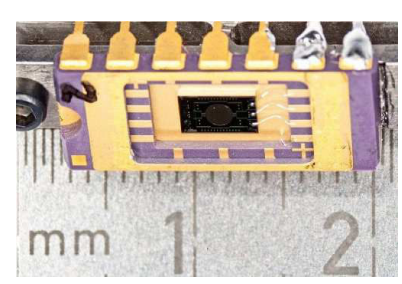

(a) MEMS mirror in DIP14

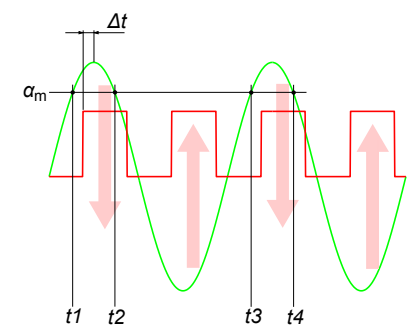

(b) MEMS driving principle and indexing measurement
Fig. 5. The MEMS mirror

a 1D mirror with approx. $250 \mathrm{~Hz}$ oscillating frequency from Hiperscan was chosen ${ }^{5}$ as depicted in Fig. 5(a). The deflection (max. $19^{\circ}$ mechanical) is driven by electrostatic forces applied by comb electrodes.

Since today there is no integrated and continuous deflection measurement available, which covers the needs of the deduced $0.06 \mathrm{mrad}$ precision, a motion model is used to estimate the actual scan angle: The mirror forms a driven harmonic oscillator with asymmetric force application, since the electrostatic effect acts always in one direction (see Fig. 5(b)): Considering a cosine movement, force can be applied only in the 1st and 3rd quadrant of the period towards the zero-position. The electric drive signal therefore is a square wave with twice the mechanic oscillating frequency. Thus the movement of the mirror $\alpha(t)$ is constrained as follows:

$$
\alpha(t)=-\alpha(t+T / 2)
$$

The oscillation can be approximated by a Fourier series as

$$
\begin{array}{r}
\alpha=\sum_{k} A a_{k} \cos \left(2 \pi k f_{0} t-\varphi_{k}-k \varphi_{0}\right) \\
\text { with } \begin{array}{l}
k=\{1,3,5,7,9,11\} \\
\varphi_{1}=0, a_{1}=1
\end{array},
\end{array}
$$

containing all odd harmonics up to the 11th and satisfying (11). This model proved to be precise enough after a FFT analysis and experiments. However, amplitude and phase of the first harmonic change significantly with the environmental conditions (e.g. temperature, humidity) due to changes in the natural frequency. Experiments showed up to $20 \%$ amplitude change from switching on to a stationary state in the laboratory. This demands closed loop operation. Therefore $A$ and $\varphi_{0}$ are measured by an indexing photodiode (see Sec. IV-D) to allow for amplitude control and synchronized scan angle calculation. The relative descriptors of the higher harmonics depend slightly from the natural frequency and amplitude and have to be calibrated for each individual mirror (see Sec. V).

\section{MEMS Indexing}

The scan angle indexing measurement is performed as depicted in Fig. 4. Contrary to related approaches [13] [14] only one index mark is needed: The MEMS mirror deflects the laser beam, which passes the scan head window and is reflected there partially. When the reflected beam passes the

\footnotetext{
${ }^{5}$ MEMS model D15B0.25 (www.hiperscan.com)
} 


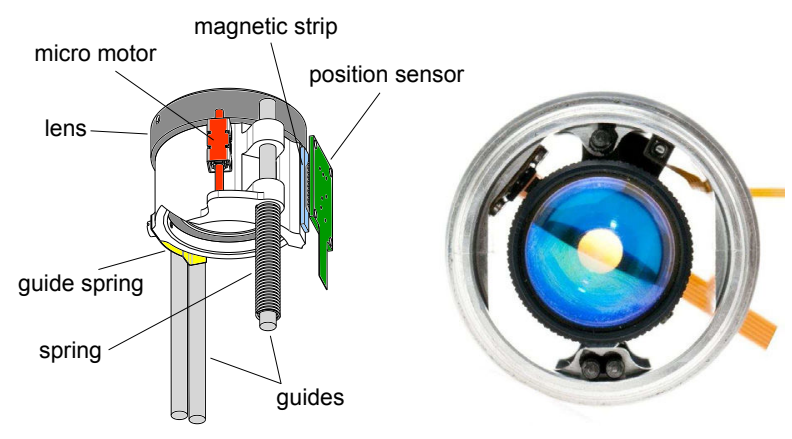

Fig. 6. Autofocus design (without housing, top view)

indexing photo diode, their two active fields produce two successive pulses, whose intersection delivers the exact and reproducible instant of time independent from laser power and environment illumination. With the required $0.06 \mathrm{mrad}$ precision and $250 \mathrm{~Hz}$ scan frequency the time resolution has to be better than $100 \mathrm{~ns}$. The phasing of the measured pulses to the MEMS driving signal ( $\Delta t$ in Fig. 5(b)) measures the natural frequency of the mirror. The time difference of two adjacent pulses $t_{n}$ yields the scan amplitude, which is controlled to a constant value. The error variable serves as a detector for mechanical shock and yields a confidence value regarding the scan angle estimation. The latter is performed knowing the absolute values of the index angular position $\alpha_{m}$ and the MEMS Fourier parameters of the motion model (12), see also Sec. V.

\section{E. Mechanics}

The mechanical design is shown in Fig. 4 and 1. The measurement is very sensitive to scan angle errors, resulting from rotational displacement of the mirror mount or camera, or translational displacement of the MEMS indexing diode. The position of the latter has to stay within $0.9 \mu \mathrm{m}$ laterally, which is hardly achieved with an aluminium construction due to thermal expansion. Therefore the scan head parts are made of Invar FeNi36 alloy, notable for a very low coefficient of thermal expansion. All other parts are produced out of aluminium alloy, since the camera housing is symmetrically round and translational expansion of the frame doesn't take measurable effect.

The autofocus is driven by a piezoelectric micro motor pushing the lens holder against a spring, see Fig. 6. The lens holder is guided free from play due to the pre-load of the guide spring. The magnetic position sensor ${ }^{6}$ yields $0.5 \mu \mathrm{m}$ resolution. The travel of the focus is designed to be $3 \mathrm{~mm}$.

\section{F. Electronics}

The indexing electronics is implemented with a two-field photo diode as follows: With equality of the two photo currents a comparator output changes polarity and so indicates that the sweeping laser beam meets exactly the transition of one photodiode field to the other one. This method is insensitive to the absolute quantity of light, since only the relative difference of the two photo currents is evaluated. To prevent uncontrolled switching of the comparator when

\footnotetext{
${ }^{6}$ Squiggle motor and Tracker position sensor from Newscale Technologies (www.newscaletech.com)
}

dark, it is activated only if the laser beam runs into one of the two photodiode fields. The threshold for this recognition is determined by a peak detector from the amplitudes of the preceding current pulses and is thus likewise independent of the absolute quantity of light. The control and evaluation of the index circuit is done in the FPGA.

\section{G. FPGA Implementation}

The following functions are implemented and tested on a FPGA evaluation board ${ }^{7}$. This implies the peak detection, the MEMS controlling and the position estimation, as well as the communication with integrated actors and sensors. The whole algorithm is designed as pipeline structure with a continuous data flow. FPGAs combine flexibility, parallelism and interconnectivity combined with high clock frequencies. Due to this FPGAs represent the most dedicated solution to implement the algorithms as well as the communication on a single chip.

1) Peak Detection: The peak detection algorithm comprises the following three stages in the pipeline structure:

Pre-work: The incoming CMOS image data is stored line by line. The maximum, average and variance of each row is calculated online during read out. The $\mathrm{S} / \mathrm{N}$ is determined and the peak can be isolated for further processing.

Peak isolation: Each peak is cut above a certain threshold depending on the noise level. At the same time the peak is checked for validation and flagged (Below $\mathrm{S} / \mathrm{N}$ threshold, invalid peak width, peak at CMOS edge).

Subpixel detection: Finally the the exact peak position is calculated in sub pixel accuracy following (8). The sine and cosine values are gathered from a quarter wave LUT. The atan 2 which leads to the exact peak position is calculated by a CORDIC in circular vectoring mode [15].

2) MEMS controlling and angle estimation: The MEMS mirror is controlled by frequency and amplitude. To start up the mirror oscillation a down sweep sequence from a high frequency is necessary. When the fixed working frequency is reached the mirrors amplitude is controlled to a constant value. Therefore the indexing time stamps are captured (cf. Sec. IV-D) and phase and amplitude are evaluated. As the best trade off between processing time and logic resources trigonometric functions and divisions are avoided and precalculated LUTs are used.

\section{CALibration AND REsults}

\section{A. Experimental Setup}

The VR-SCAN used in the following experiments consists of the complete mechanical and optical prototype, an offthe-shelf Camera Link line scan camera body containing the selected CMOS sensor (Fairchild OWL), a PC with frame grabber, the FPGA evaluation board and the MEMS indexing PCB (see Fig. 7). The image processing takes place offline in the PC, the FPGA synchronizes the MEMS and the camera and does the indexing processing. The mirror is amplitude controlled. Unless noted otherwise the mirror runs at a stable stationary operating point.

The scanner is mounted on a positioning stage in front of a linear stage in $\mathrm{z}$ - direction (5 $\mu \mathrm{m}$ resolution), which is used for exact translation of the calibration surface.

\footnotetext{
${ }^{7}$ ML506 Development platform with Virtex-5 SX50 (www.xilinx.com)
} 


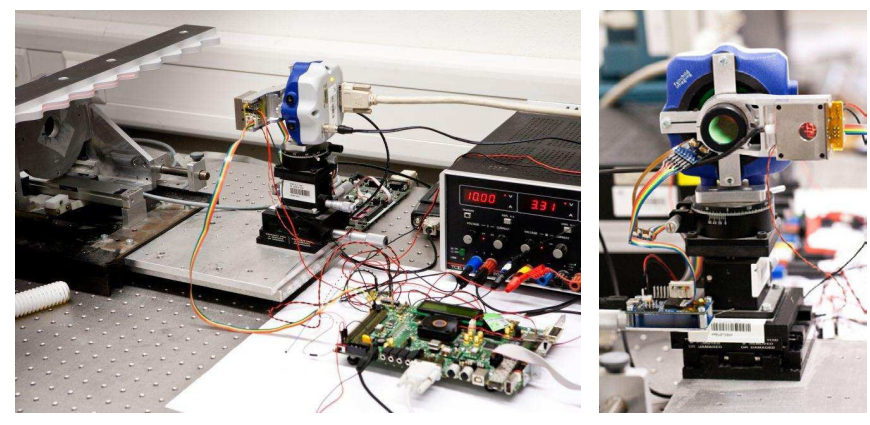

Fig. 7. The experimental setup

\section{B. Calibration procedure}

Equation (3) delivers the transformation from sensor to cartesian coordinates. With (12) the sensor model is complete. Hence 5 geometric and 2 lens distortion parameters, 10 mirror motion harmonics descriptors and the MEMS indexing measuring position are to be calibrated. The measured variables are $x_{s}$, and the mirrors base oscillation phase and amplitude delivered by the indexing device. However, the MEMS movement has to be measured continuously in order to determine the Fourier descriptors of the motion model. This is achieved best by the fast but also uncalibrated CMOS camera itself, ending up in a chicken and egg dilemma. This is resolved by the following procedure:

1) Coarse calibration of the complete sensor by minimizing all 18 parameters by scanning a known depth pattern (relief) at multiple known distances with fixed focus

2) Fine calibration of the MEMS mirror with the calibrated camera by scanning a known plane at different operating points

3) LUT generation over the whole measuring range and different focus positions with known scan angles by scanning a known plane

In the following, each "scan" consists of 10000 consecutive frames at $37.4 \mathrm{kHz}(150.1$ times the scan frequency of $249.0 \mathrm{~Hz}$ ). This features 10-fold scan angle interlacing. The laser power is kept constant in all scans. In the first step a known sine relief serves as ground truth and is scanned at multiple distances. The depth pattern is mounted on a sled whose relative z-position is measured at high resolution (see Fig. 7). Thus the geometric parameters are well-conditioned. The model parameters are varied such that the sum of all errors in z-direction is minimized using a simplex search algorithm. Distortions due to de-focus and laser spot asymmetries are not considered. Fig. 8 depicts the result of this step. The error averages to $0.30 \mathrm{~mm}$.

The second step uses the roughly calibrated camera to analyze the mirrors parameters at different (amplitude|frequency) operating points. Therefore a plane rectangular to the $\mathrm{z}$-axis at known distance is scanned. Only the mirror parameters are optimized for a minimal z-error sum. This procedure yields a mirror calibration map allowing for both multiple operating points and natural frequency drift due to temperature instability. The primarily changing value is the phase, the (relative) motion model harmonic parame-

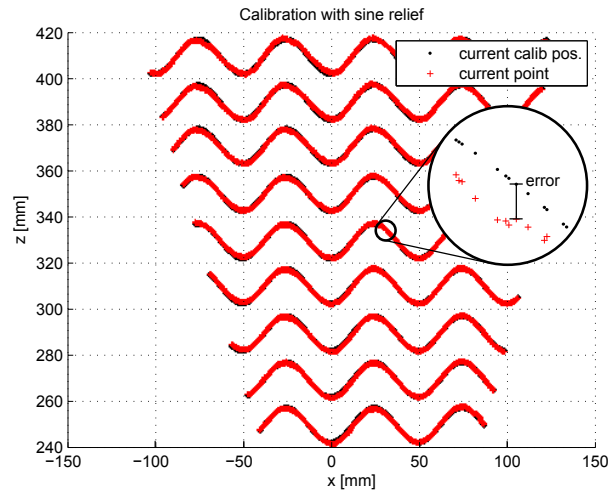

Fig. 8. Calibration with sine depth pattern

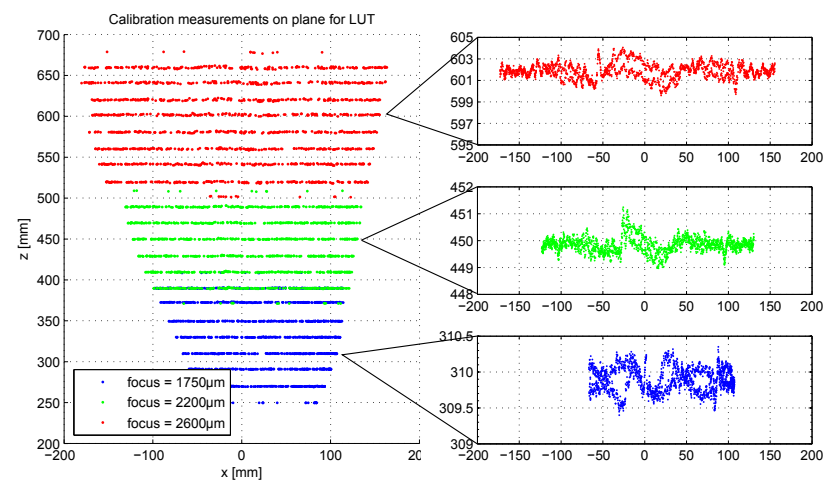

Fig. 9. Calibration scans on plane for LUT generation

ters roughly stay constant. However, a slight dependency on the phase is observed.

Finally, the plane is scanned multiple times covering the whole measuring field at different discrete focus settings with overlapping range depths. For each focus position a LUT is generated for the conversion to cartesian coordinates. Doing so, laser spot asymmetries and focus blur are considered as well. In Fig. 9 three scan sets with error details are depicted, each at different focus.

\section{Results}

A first result is shown in Fig. 9. The plots represent the error in z-direction after static plane scans at various distances and are used for the LUT generation. The accuracy is between approx. $\pm 0.4 \mathrm{~mm}$ at close range and \pm 1.0 $\mathrm{mm}$ at far range. Thus the error is slightly greater than the demanded $0.1 \%$ range precision (see Tab. III). It is expected that the accuracy will improve when adaptive laser power is implemented. The macroscopic corrugation which is observable in the enlarged details depends from the scan angle only and is time-independent, since the point clouds consist of more than 60 overlapping MEMS scan periods. Therefore it is assumed, that it's caused by a combination of laser speckle noise and the MEMS scan angle estimation residual error.

To confirm the calibration procedure a threaded bolt was scanned in arbitrary orientations and distances at three different focus positions (see Fig. 10). Except for simple S/N 
TABLE III

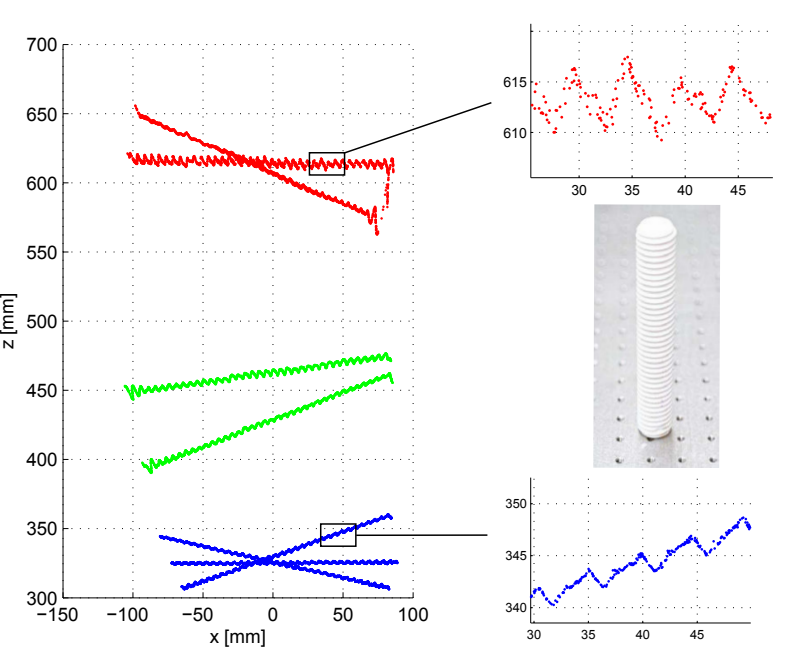

Fig. 10. Test scan of a threaded bolt at arbitrary orientations
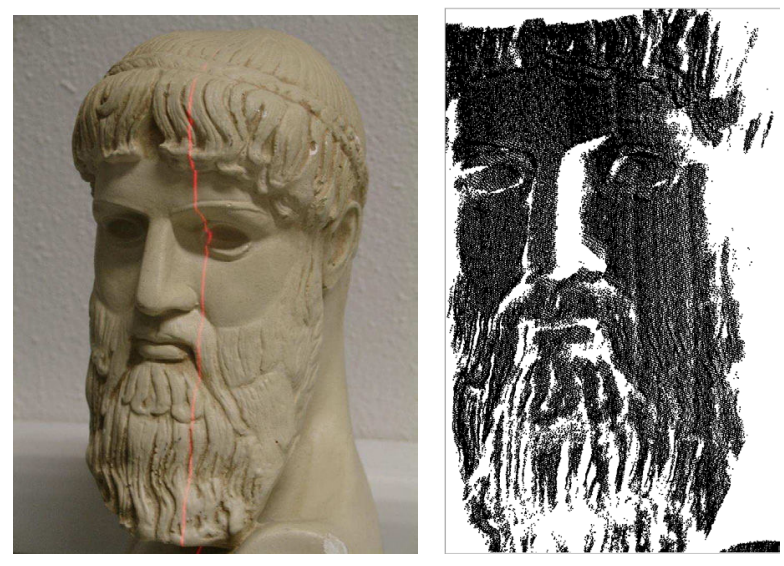

Fig. 11. Sample 3D point cloud of a Zeus bust

filtering the raw data is depicted. The detail enlargements show the thread pitch of $5 \mathrm{~mm}$ even at $600 \mathrm{~mm}$ distance. However, some distortions are observed, which derive from speckle noise and the projected laser spot asymmetry. Fig. 11 shows a sample point cloud of a Zeus bust, consisting of 280966 points acquired in one linear pan over the object.

Tab. III summarizes the scanners specifications, including the design goals if differing.

\section{CONCLUSION}

This work presents the prototype implementation of VRSCAN. Its design aims on robust and versatile $3 \mathrm{D}$ data acquisition in a lightweight and small package. Optical, mechanical and electronics design was aligned to reach this goal. The scanner was built in an experimental setup and successfully calibrated. First measurements confirm this approach. In near future, adaptive laser power and focus control will be implemented. Further, the CMOS will be included in the FPGA design replacing the CameraLink camera. After all, an integrated electronics design will permit stand alone operation on the robot. In respect of the multi sensory approach [1] it is planned to augment the VR-SCAN
SPECIFICATIONS OF VR-SCAN

\begin{tabular}{llll}
\hline Measuring depth & 750 & $\mathrm{~mm}$ & with auto focus \\
Measuring distance & $250-1000$ & $\mathrm{~mm}$ & with auto focus \\
Scan width & $140-570$ & $\mathrm{~mm}$ & near - far \\
Measuring data rate & 38 & $\mathrm{kHz}$ & \\
Point density & $150(1500)$ & points/line & (interlaced) \\
Avg. angular res. & $9(0.9)$ & $\mathrm{mrad}$ & (interlaced) \\
& 1.7 & $\mathrm{mrad}$ & design goal \\
Accuracy & $\pm 0.2(0.1)$ & $\%$ of range & (design goal) \\
Dimensions & $125 \times 80 \times 50$ & $\mathrm{~mm}$ & \\
Weight & $\approx 400$ & $\mathrm{~g}$ & complete \\
\hline
\end{tabular}

with cameras to allow for further applications like handguided and mobile operation such as in medicine, CSI and cultural heritage preservation.

\section{ACKNOWLEDGEMENT}

The authors wish to thank Tim Bodenmüller and Ulrich Hagn for their support and helpful discussions.

\section{REFERENCES}

[1] M. Suppa, S. Kielhöfer, J. Langwald, F. Hacker, K. Strobl, and G. Hirzinger, "The 3d-modeller: A multi-purpose vision platform," in Proc. of ICRA, April 2007, pp. 781-787.

[2] S. Fuchs, S. Haddadin, M. Keller, S. Parusel, A. Kolb, and M. Suppa, "Cooperative bin-picking with time-of-flight camera and impedance controlled dlr lightweight robot iii," in Proc. of IROS, Oct. 2010, pp. $4862-4867$.

[3] A. Schwier, R. Konietschke, T. Bodenmüller, T. Ende, S. Kielhöfer, and G. Hirzinger, "Vr-map: A new device for patient registration and optimal robot positioning," in CURAC, 2010.

[4] M. Suppa, "Autonomous robot work cell exploration using multisensory eye-in-hand systems," Ph.D. dissertation, Universität Hannover, 2007.

[5] F. Blais, "Review of 20 years of range sensor development," Journal of Electronic Imaging, vol. 13(1), pp. 231-243, 2004.

[6] K. Strobl, W. Sepp, E. Wahl, T. Bodenmüller, M. Suppa, J. Seara, and G. Hirzinger, "The dlr multisensory hand-guided device: The laser stripe profiler," in Proc. of ICRA, vol. 2, 26-May 1, 2004, pp. 19271932 Vol.2.

[7] H. Kawata, A. Ohya, S. Yuta, W. Santosh, and T. Mori, "Development of ultra-small lightweight optical range sensor system," in Proc. of IROS, Aug. 2005, pp. 1078-1083.

[8] F. Hacker, J. Dietrich, and G. Hirzinger, "A laser-triangulation based miniaturized 2-d range-scanner as integral part of a multisensory robotgripper." in Proceedings EOS 14th Topical Meeting on Optoelectronic Distance/ Displacement Measurements and Applications. Ecole des Mines de Nantes, France, 1997.

[9] M. Rioux, G. Bechthold, D. Taylor, and M. Duggan, "Design of a large depth of view three-dimensional camera for robot vision," Optical Engineering, vol. 26, no. 12, pp. 1245-1250, 1987.

[10] Z. Zhang, "A flexible new technique for camera calibration," Pattern Analysis and Machine Intelligence, IEEE Transactions on, vol. 22, no. 11 , pp. 1330-1334, 2000.

[11] R. B. Fisher and D. K. Naidu, "A comparison of algorithms for subpixel peak detection," in Image Technology, Advances in Image Processing, Multimedia and Machine Vision, 1996, pp. 385-404.

[12] B. Fowler, J. Balicki, D. How, S. Mims, J. Canfield, M. Godfrey, and P. Inc, "An ultra low noise high speed cmos linescan sensor for scientific and industrial applications," in Proc. Workshop on CCD and AIS, Elmau, Germany, 2003.

[13] A. Wolter, H. Schenk, H. Korth, and H. Lakner, "Torsional stress, fatigue and fracture strength in silicon hinges of a micro scanning mirror," in Proceedings of SPIE, D. M. Tanner and R. Ramesham, Eds., vol. 5343, 2004, pp. 176-185.

[14] A. Tortschanoff, M. Lenzhofer, A. Frank, M. Wildenhain, T. Sandner, H. Schenk, and A. Kenda, "Optical position feedback and phase control of moems scanner mirrors," in Proceedings of SPIE, vol. 7594, 2010, p. $75940 \mathrm{~K}$.

[15] R. Andraka, "A survey of cordic algorithms for fpga based computers," in Proceedings of the 1998 ACM/SIGDA sixth international symposium on Field programmable gate arrays. ACM, 1998, pp. 191-200. 